\title{
$\mathrm{g}^{\circ}$
}

\section{Peierls Substitution in an Engineered Lattice Potential}

\author{
K. Jiménez-García, ${ }^{1,2}$ L. J. LeBlanc, ${ }^{1}$ R. A. Williams, ${ }^{1}$ M. C. Beeler, ${ }^{1}$ A. R. Perry, ${ }^{1}$ and I. B. Spielman ${ }^{1, *}$ \\ ${ }^{1}$ Joint Quantum Institute, National Institute of Standards and Technology and University of Maryland, \\ Gaithersburg, Maryland, 20899, USA \\ ${ }^{2}$ Departamento de Física, Centro de Investigación y Estudios Avanzados del Instituto Politécnico Nacional, \\ México D.F., 07360, México \\ (Received 31 January 2012; published 29 May 2012)
}

\begin{abstract}
Artificial gauge fields open the possibility to realize quantum many-body systems with ultracold atoms, by engineering Hamiltonians usually associated with electronic systems. In the presence of a periodic potential, artificial gauge fields may bring ultracold atoms closer to the quantum Hall regime. Here, we describe a one-dimensional lattice derived purely from effective Zeeman shifts resulting from a combination of Raman coupling and radio-frequency magnetic fields. In this lattice, the tunneling matrix element is generally complex. We control both the amplitude and the phase of this tunneling parameter, experimentally realizing the Peierls substitution for ultracold neutral atoms.
\end{abstract}

DOI: 10.1103/PhysRevLett.108.225303

Ultracold atoms subjected to artificial gauge fields can realize phenomena usually in the domain of electronic systems. Prime examples include the quantum Hall effect (for Abelian gauge fields), and topological insulators (for non-Abelian gauge fields) [1]. Many of these phenomena are predicted to occur at extremely low temperatures, and adding a lattice potential to an ultracold system can increase the energy scales at which strongly correlated states are expected to emerge [2,3]. Current techniques for generating periodic potentials in ultracold atom systems use optical standing waves created with suitably polarized counterpropagating lasers [4]. In contrast, we describe a one-dimensional (1D) "Zeeman lattice" for ultracold atoms created with a combination of radio frequency (rf) and optical-Raman coupling fields, without any optical standing waves. In this lattice, atoms acquire a quantum mechanical phase as they hop from site to site, explicitly realizing the Peierls transformation [5] in the laboratory frame. Our approach extends existing Raman dressing schemes [6] by simultaneously generating an artificial gauge field and an effective lattice potential.

Optical lattices generally result from the electric dipole interaction between an atom and the electric field of an optical standing wave, yielding a potential $V_{\text {dip }}(\mathbf{r}) \propto$ $\alpha(\lambda) \mathbf{I}(\mathbf{r})$, where $\alpha(\lambda)$ is the atomic polarizability at the laser wavelength $\lambda$, and $I(\mathbf{r})$ is the spatial intensity distribution [4]. In such lattices, the natural units of momentum and energy are given by the single photon recoil momentum $\hbar k_{L}=2 \pi \hbar / \lambda$ and its corresponding energy $E_{L}=$ $\hbar^{2} k_{L}^{2} / 2 m$, where $m$ is the atomic mass.

Published by the American Physical Society under the terms of the Creative Commons Attribution 3.0 License. Further distribution of this work must maintain attribution to the author(s) and the published article's title, journal citation, and DOI.
PACS numbers: 67.85.Fg, 03.65.Vf, 03.75.Lm, 67.90.+z

Quantum particles with charge $q$ in a 1D periodic potential (here along $\mathbf{e}_{x}$ ) acquire a phase $\phi_{j}=(q / \hbar) \int_{x_{j}}^{x_{j+1}} \mathbf{A}$. $\mathbf{e}_{x} d x$ upon tunneling from site $j$ to $j+1$ in the presence of a vector potential $\mathbf{A}$. For sufficiently strong lattice potentials, this system is described by the tight-binding Hamiltonian

$$
H=-\sum_{j}\left[t \exp \left(i \phi_{j}\right) \hat{a}_{j+1}^{\dagger} \hat{a}_{j}+\text { H.c. }\right],
$$

where $\hat{a}_{j}^{\dagger}$ describes the creation of a particle at site $j$, and $t \exp \left(i \phi_{j}\right)$ is the complex matrix element for tunneling between neighboring sites. Using the phases $\phi_{j}$ to represent the effect of $\mathbf{A}$ is known as the Peierls substitution [5], and for a uniform phase $\phi$ the energy is $E\left(k_{x}\right)=$ $-2 t \cos \left(\pi k_{x} / k_{L}-\phi\right)$, where $k_{x}$ is the particle's crystal momentum.

We realize the Peierls substitution for ultracold atoms by synthesizing a 1D effective Zeeman lattice that allows independent control of both $t$ and $\phi$. Previous experiments (a) controlled the amplitude and sign of $t$ in driven optical lattices [7], or, in addition, (b) controlled $\phi$ by means of rotating optical lattices [8] or Raman-assisted tunneling in an optical superlattice [9]. Our effective Zeeman lattice provides both a periodic potential and an artificial vector potential in the laboratory frame.

The Zeeman lattice arises from a combination of $\mathrm{rf}$ and Raman fields that simultaneously couple the spin states $\left\{\left|m_{F}\right\rangle\right\}_{m_{F}=0, \pm 1}$ of a ${ }^{87} \mathrm{Rb}$ Bose-Einstein condensate (BEC) in the $F=1$ ground level, which are split by $\hbar \omega_{Z}$ [Fig. 1(a) and 1(b)]. In the frame rotating at the rf frequency $\Delta \omega$ and under the rotating wave approximation, the combined rfRaman coupling contributes a term,

$$
\hat{H}_{\mathrm{rf}+R}(x)=\boldsymbol{\Omega}(x) \cdot \hat{\mathbf{F}}+\hat{H}_{Q},
$$

to the overall Hamiltonian, where $\hat{\mathbf{F}}=\left(\hat{F}_{x}, \hat{F}_{y}, \hat{F}_{z}\right)$ is the $F=1$ angular momentum operator; $\boldsymbol{\Omega}=\left(\Omega_{\mathrm{rf}}+\right.$ $\left.\Omega_{R} \cos \left(2 k_{L} x\right),-\Omega_{R} \sin \left(2 k_{L} x\right), \sqrt{2} \delta\right) / \sqrt{2}$, in which $\Omega_{\mathrm{rf}}$ 
(a) Level diagram

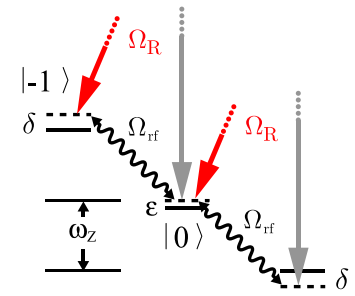

(b) Experimental setup

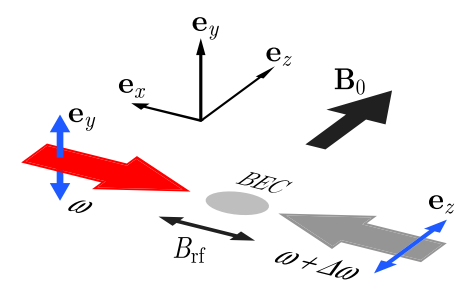

$|+1\rangle$

(c) Effective Zeeman lattice

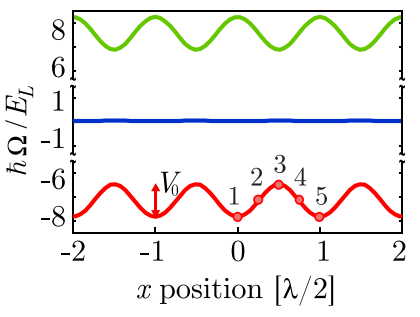

(d) Peierls phase

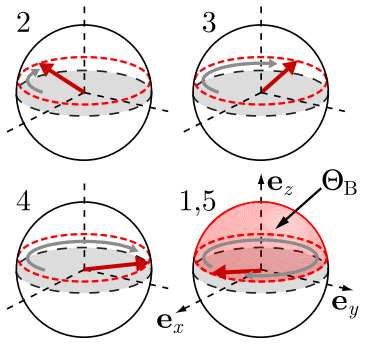

FIG. 1 (color online). Effective Zeeman lattice. (a)-(b) A uniform magnetic field $B_{0} \mathbf{e}_{y}$ Zeeman splits the levels in the $F=1$ ground state manifold of ${ }^{87} \mathrm{Rb}$ by $\omega_{Z}$ and provides a quadratic Zeeman shift $\epsilon$. In conjunction with a rf magnetic field $B_{\mathrm{rf}} \mathbf{e}_{x}$ with frequency $\Delta \omega$, a pair of orthogonally polarized counterpropagating Raman beams with frequencies $(\omega, \omega+\Delta \omega)$ illuminates the atomic sample. The rf and Raman fields have coupling strengths $\Omega_{\mathrm{rf}}$ and $\Omega_{R}$. (c) The spatially varying eigenvalues of $\hat{H}_{\mathrm{rf}+R}(x)$ (red, blue, and green curves) give rise to our $\lambda / 2$ effective Zeeman lattice, as plotted $\hbar\left(\Omega_{\mathrm{rf}}, \Omega_{R}, \delta\right)=$ $(1,10,2) E_{L}$. (d) Spatial precession of $\mathbf{B}_{\text {eff }}(x)$ (dark arrow) and the solid angle it subtends when an atom tunnels to the nearest neighboring site [points $1-5$ in (c)]. This geometrical Berry's phase $\Theta_{B}$ gives the Peierls phase $\phi$. Only when both dressing fields are illuminating the atoms does the lattice potential exist; therefore, the effective Zeeman field is, in general, not symmetric about $\mathbf{e}_{z}$.

and $\Omega_{R}$ are the rf and Raman coupling strengths, $\delta=$ $\Delta \omega-\omega_{Z}$ is the detuning from Raman resonance, and $H_{\mathrm{Q}}=-\epsilon\left(\hbar^{2} \hat{1}-\hat{F}_{z}^{2}\right) / \hbar$ describes the quadratic Zeeman shift. Equation (2) is the Zeeman Hamiltonian for an effective field $\mathbf{B}_{\text {eff }}(x)=\hbar \boldsymbol{\Omega}(x) / g_{F} \mu_{B}$, where $\mu_{B}$ is Bohr's magneton and $g_{F}$ is Landé's $g$ factor. This spatially varying effective Zeeman shift produces a $1 \mathrm{D}$ lattice potential [Fig. 1(c)]. As atoms tunnel from site to site, $\mathbf{B}_{\text {eff }}$ spatially precesses [Fig. 1(d)] and the atoms acquire a geometrical Berry's phase $\Theta_{B}$ [10] proportional to the enclosed solid angle. The tunneling parameters $t$ and $\phi$, obtained from $E\left(k_{x}\right)$, are nontrivial functions of $\boldsymbol{\Omega}$. In the limit of large Raman coupling $\left(\Omega_{R} \gg 4 E_{L}, \Omega_{\mathrm{rf}}, \epsilon\right)$, the Peierls phase is $\phi=\Theta_{B}+2 \pi$, where the Berry's phase is $\Theta_{B}=2 \pi m_{F}(1-\delta / \Omega)$, with $m_{F}=-1$ and $\Omega=$ $\left(\Omega_{R}^{2} / 2+\delta^{2}\right)^{1 / 2}$, independent of $\Omega_{\mathrm{rf}}$. In our experiment, $\Omega_{R}$ is not sufficiently large for this approximation to be valid, so we instead compare our results to the numerically computed band structure.
When $\Omega_{\mathrm{rf}} \gg \Omega_{R}, \delta, \epsilon$ the effective Zeeman shift reduces to $\hbar|\Omega| \approx \hbar\left[\Omega_{\mathrm{rf}}+\Omega_{R} \cos \left(2 k_{L} x\right)\right] / \sqrt{2}$, and when $\Omega_{R} \gg \Omega_{\mathrm{rf}}, \delta$ we obtain the analogous result $\hbar|\boldsymbol{\Omega}| \approx$ $\hbar\left[\Omega_{R}+\Omega_{\mathrm{rf}} \cos \left(2 k_{L} x\right)\right] / \sqrt{2}$. In both of these limits, the larger of the two fields defines a natural quantization axis about which the smaller field spatially modulates $|\Omega|$. For $\Omega_{R} \gg \Omega_{\mathrm{rf}}$, this quantization axis is spatially rotating.

We experimentally characterize the lattice in three ways: (i) we measure the effective mass $m^{*}=$ $\hbar^{2}\left[d^{2} E\left(k_{x}\right) / d k_{x}^{2}\right]^{-1}$, which in the tight-binding regime is inversely proportional to $t$; (ii) we quantify the Peierls phase $\phi$ and test its robustness against small changes in $\Omega_{\mathrm{rf}}$; and (iii) we investigate the diffraction of BECs from our effective Zeeman lattice. In each case, we start with ${ }^{87} \mathrm{Rb} \mathrm{BECs}$ in the $\left|F=1, m_{F}=-1\right\rangle$ state in a crossed optical dipole trap with frequencies $\left(f_{x}, f_{y}, f_{z}\right)=$ $(13,45,90) \mathrm{Hz}$ [11]. In the presence of a uniform bias field $B_{0} \mathbf{e}_{y}$, we apply a rf magnetic field with frequency $\Delta \omega / 2 \pi=g_{F} \mu_{B} B_{0}=3.25 \mathrm{MHz}$ and prepare the BEC in the lowest energy rf-dressed state [12]. Two $\lambda=$ $790.33 \mathrm{~nm}$ Raman laser beams, counter-propagating along $\mathbf{e}_{x}$ and differing in frequency by $\Delta \omega$, couple the BEC's internal degrees of freedom with strength $\Omega_{R}$ [Fig. 1(a) and 1(b)]. The combination of rf and Raman coupling creates a 1D lattice potential along $\mathbf{e}_{x}$, the direction of momentum exchange defined by the Raman beams.

We obtain the atoms' effective mass $m^{*}$ by inducing dipole oscillations [13-15] along $\mathbf{e}_{x}$ and measuring shifts in the oscillation frequency as a function of the coupling strengths $\Omega_{R}$ and $\Omega_{\mathrm{rf}}$. The atoms slosh in the lattice for a variable time $\tau$, after which we remove all coupling and confining potentials (thus projecting the final spinmomentum superposition into bare atomic states) and absorption image the atoms after a $28.2 \mathrm{~ms}$ time-of-flight (TOF). Figure 2(a) shows bare and dressed condensates oscillating at frequencies $f_{x}$ and $f^{*}$, respectively [16]; Fig. 2(b) shows that the effective to bare mass ratio $m^{*} / m=\left(f_{x} / f^{*}\right)^{2}$, as a function of $\Omega_{R}$ and $\Omega_{\mathrm{rf}}$, is in good agreement with calculations (curves [14]). These data provide the tunneling matrix element amplitude $t / E_{L}=\left(\mathrm{m} / \mathrm{m}^{*}\right) / \pi^{2}$ in the tight-binding regime [17].

An important characteristic of our effective Zeeman lattice is the presence of a tunable Peierls hopping phase $\phi$, which can be revealed through its effects on $E\left(k_{x}\right)$ and is experimentally controlled by adjusting $\Omega_{z}$. We identify $\phi$ both by adiabatically modifying the band structure ("adiabatic method") and by inducing oscillations ("sudden method," similar to above). Furthermore, we test its insensitivity to variations in $\Omega_{\mathrm{rf}}$ with the latter method.

In the adiabatic method, we load a BEC at $k_{x}=0$ and adiabatically change $\Omega_{z}$, such that the BEC always sits at the minimum of $E\left(k_{x}\right)$ located at $k_{\min }$. The time scale for adiabaticity is set by the modified trapping frequency $f^{*}$ along the direction of the Raman beams. Once $\Omega_{z}$ reaches its final value, we remove the trapping potential and 
(a) Induced sloshing on bare $\square$ and dressed BECs $\odot$

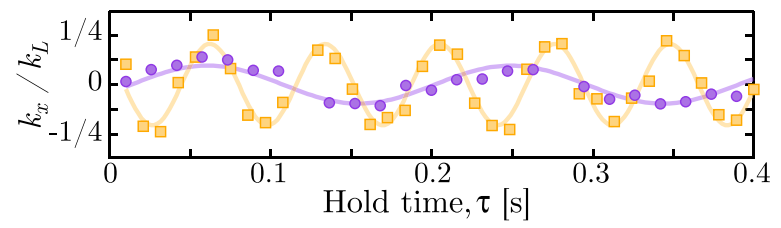

(b) Effective mass

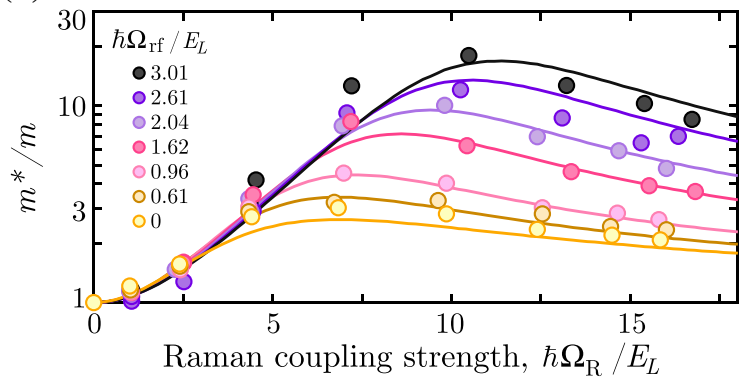

FIG. 2 (color online). Effective mass. (a) Comparison of the oscillations of a BEC in the $\left|m_{F}=-1\right\rangle$ state to those in a rfRaman-dressed BEC $\left[\hbar \Omega_{R}=12.4(9) E_{L}\right.$ and $\hbar \Omega_{\mathrm{rf}}=2.04(6)$ $\left.E_{L}\right]$. The curves are fits to a sinusoid from which we obtain $f_{x}=14.0(1) \mathrm{Hz}$ and $f^{*}=5.3(1) \mathrm{Hz}$, thus $m^{*} / m=7.0(3)$ and $t=0.015(1) E_{L}$. (b) Measurements of $m^{*} / m$ as a function of $\Omega_{R}$ and $\Omega_{\mathrm{rf}}$. The curves depict the expected $m^{*} / m$ ratio.

subsequently deload all atoms into the $\left|m_{F}=+1\right\rangle$ spin state while mapping the occupied crystal momentum $k_{x}$ to free-particle momentum [14]. We image this distribution after a $13.1 \mathrm{~ms}$ TOF, revealing $k_{\text {min }}$. The Peierls phase, shown as crosses in Fig. 3(a), is $\phi / \pi=k_{\min } / k_{L}$.

In the sudden method, we test the robustness of the Peierls phase $\phi$ by first adiabatically loading to $\phi= \pm \pi$ (the condensate sits at the edge of the Brillouin zone) and then suddenly changing both $\Omega_{z}$ and $\Omega_{\mathrm{rf}}$ [18] to new values (changing both $\phi$ and $t$ ). This results in momentum space oscillations centered at $k_{\min }$. After a time $\tau$ we release the BEC, and measure as above. We fit the crystal momentum dynamics with $k_{x}(\tau)=k_{\min }+\Delta k_{x} \cos \left(2 \pi \tau f^{*}+\gamma\right)$, where $\Delta k_{x}$ is the amplitude, and $\gamma$ is an overall phase shift whose average value is $0.9(1) \pi$ for these measurements [14]. Figure 3(a) (circles) shows the measured Peierls tunneling phase as a function of $\Omega_{z}$.

Measurements from the adiabatic and sudden methods are in good agreement with each other and their expected values [Fig. 3(a), dashed curves], highlighting the precise experimental control offered by our rf-Raman induced effective Zeeman lattice. This agreement also demonstrates the robustness of our engineered Hamiltonian to deliberate variations in $\Omega_{\mathrm{rf}}$ of up to $0.25 E_{L}$, as was anticipated by the absence of $\Omega_{\mathrm{rf}}$ in the large $\Omega_{R}$ expression for $\phi$. We find that the hopping phase is unaltered by small changes in $\Omega_{\mathrm{rf}}$ even when $t$ changes significantly.

The sloshing amplitude $\left|\Delta k_{x}\right|$ is displayed in Fig. 3(b). For large initial $\left|\Delta k_{x}\right|$ (shaded region) we observe the depletion of BEC atoms and a strong damping of the center of mass oscillation (evident from the departure of the (a) Peierls tunneling phase

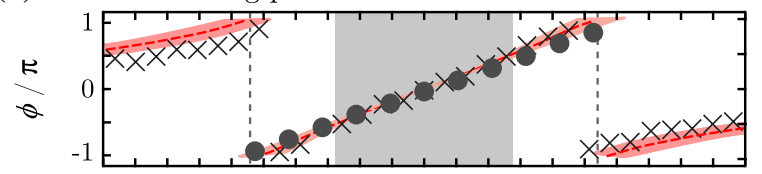

(b) Sloshing amplitude

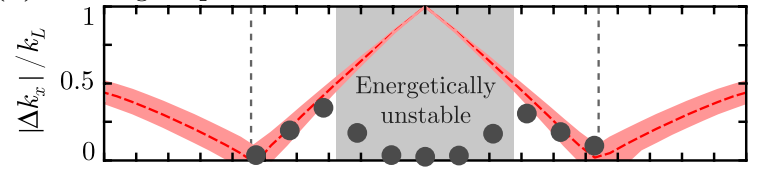

(c) Tunneling amplitude

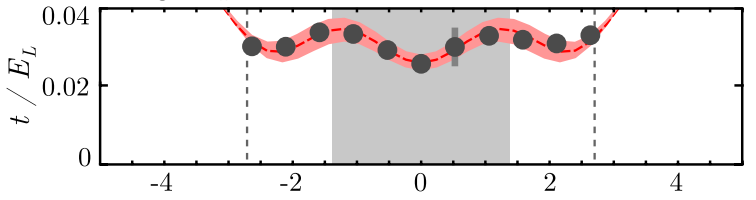

Effective Zeeman field along $z, \hbar \Omega_{z} / E_{L}$

FIG. 3 (color online). Peierls transformation. (a) Peierls phase $\phi$ measured using adiabatic (crosses) and sudden (circles) changes of $\Omega_{z}$. Vertical lines denote the first Brillouin zone. (b) Sloshing amplitude after suddenly changing $\Omega_{z}$. We observed strong damping of oscillations in the region shaded in gray. (c) Tunneling amplitude $t$ measured from oscillation frequency. The rf coupling was modulated as a function of $\Omega_{z}$ to test the robustness of the Peierls phase $\phi$. The Raman coupling was held at $\hbar \Omega_{R}=10.0(8) E_{L}$. The dashed curves correspond to the expected behavior calculated from $H_{\mathrm{rf}+R}$, and the pink bands arise from the experimental uncertainty in $\Omega_{R}$.

oscillation amplitude from the value predicted by singleparticle arguments). Both of these effects are signatures of an energetic instability in the dynamics of a BEC moving in a combined harmonic plus periodic potential [19]. The region of strong damping observed in our system coincides with the expected range $\Delta k_{x}>0.5 k_{L}$ (shaded gray region) of this dynamical instability [19]. Figure 3(c) displays the tunneling amplitude $t$, obtained from $f^{*}$. For comparison, a sinusoidal lattice would require a depth of $V_{0} \approx 8 E_{L}$ to give similar parameters.

Having discussed the behavior of atoms in the lattice's lowest band, we now explore the full lattice by suddenly turning it on, diabatically projecting a ground state BEC into higher bands. At the beginning of such a pulse, an ordinary periodic potential would first spatially modulate the BEC's phase before the atoms begin to move [20]; our effective Zeeman lattice induces such a modulation but in a spin-dependent manner. We focus on the $\Omega_{R} \gg \Omega_{\mathrm{rf}}$ and $\Omega_{R} \ll \Omega_{\mathrm{rf}}$ tight-binding regimes and investigate the spin and spatial structure of our lattice. Our data extends well beyond the short-time phase modulation regime.

In the absence of either Raman or rf coupling, there is no lattice. As indicated in Fig. 4(a), we use two different methods to introduce our lattice on an initial spatially uniform state: (i) starting with a rf-dressed state (with $\left.k_{x}=0\right)$, we suddenly $\left(t_{\mathrm{on}}<1 \mu \mathrm{s}\right)$ turn on the Raman 
(a) Pulse sequences

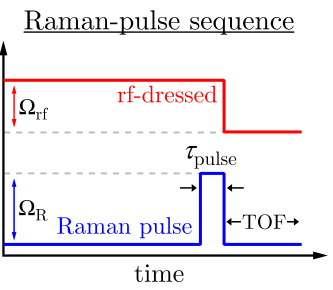

(b) Typical TOF data for large atom number

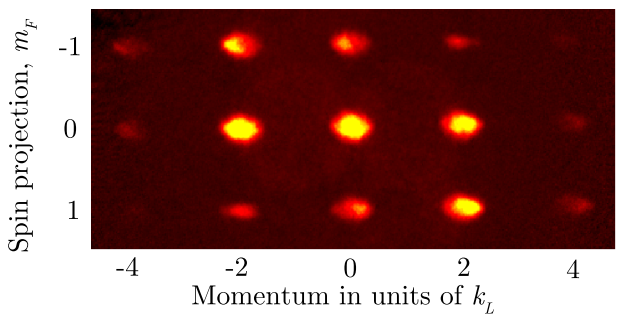

(c) rf-dressed + Raman-pulse
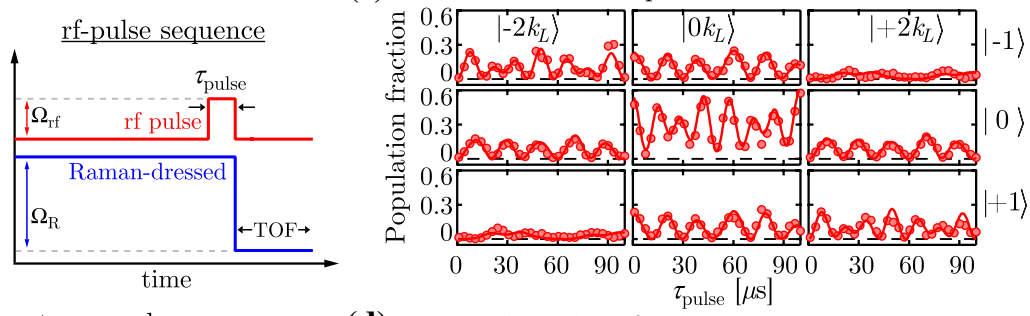

(d) Raman-dressed + rf-pulse

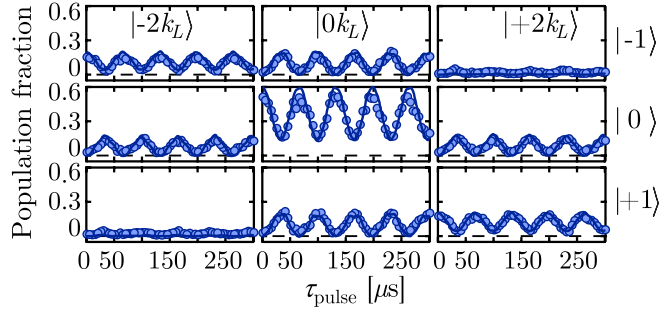

FIG. 4 (color online). BEC diffraction from the effective Zeeman lattice. (a) Starting with a rf-dressed (Raman-dressed) state, we suddenly turn on the Raman (rf) field for a variable time $\tau_{\text {pulse }}$. (b) Using TOF absorption images of the projected spin-momentum distributions, we count the number of atoms in each diffracted order and determine its fractional population. Panels (c) and (d) depict the time evolution of these fractions. The curves are fits to the data, calculated from $H_{\mathrm{rf}+R}$. The fit parameters are (c) rf dressed $\hbar\left(\Omega_{\mathrm{rf}}, \Omega_{R}, \Omega_{z}\right)=(3.57,11.49,-0.04) E_{L}$ and (d) Raman dressed $\hbar\left(\Omega_{\mathrm{rf}}, \Omega_{R}, \Omega_{z}\right)=(3.06,15.14,0.08) E_{L}$.

beams; or (ii) starting with a Raman-dressed state [12] (a superposition of $\left|m_{F}=0, k_{x}=0\right\rangle$ and $\mid m_{F}= \pm 1, k_{x} \mp$ $\left.2 k_{L}\right\rangle$ ), we suddenly turn on the rf field.

After holding the lattice on for a time $\tau_{\text {pulse }}$, we suddenly turn off the rf and Raman fields, together with the confining potential. The atoms are projected onto the bare spinmomentum basis and separate in TOF in the presence of a magnetic field gradient (along $\mathbf{e}_{z}$ ), allowing us to resolve their spin and momentum components.

We observe a detectable population in states with momenta up to $\left|k_{x}\right| \leq 4 k_{L}$ [Fig. 4(b)]. We perform such experiments for $\Omega_{R} / \Omega_{\mathrm{rf}} \approx 3$ and 5 . We minimize the effects of interactions by working with small BECs ( $\approx 9 \times 10^{4}$ atoms). Figures 4(c) and 4(d) show the fraction of atoms in each diffracted order evolving with time. We observe multiple revivals of the initial spin-momentum state and find symmetry in the population dynamics of spin-momentum states with opposite momentum and opposite spin. The curves represent fits to the populations in all spin-momentum components. The parameters from the fits are all within $10 \%$ for our calibrated values, demonstrating that the spin-momentum dynamics are well described by the unitary evolution of the initial states under $H_{\mathrm{rf}+R}$ [14].

Based on this technique for controlling the Peierls phase and inspired by recent proposals for creating flux lattices $[1,21]$, we now describe how this method might be extended to create a lattice with zero net flux that is topologically equivalent to the Hofstadter model with flux density $n_{\Phi}=1 / 3$ per plaquette. Because the hopping phase is only defined modulo $2 \pi$ (thus $n_{\Phi}$ is only defined modulo 1), a uniform magnetic field with $n_{\Phi}=1 / 2$ is equivalent to a staggered field with $n_{\Phi}= \pm 1 / 2$. In the same spirit, a magnetic field staggered along $\mathbf{e}_{z}$ with flux density $(\ldots, 1 / 3,1 / 3,-2 / 3, \ldots$,$) has zero net flux yet is$ equivalent to a uniform field with $n_{\Phi}=1 / 3$. These fields could be generated by the Peierls phases $\phi_{y}\left(j_{x}, j_{z}\right)=0$ and $\phi_{x}\left(j_{x}, j_{z}\right)=-(2 \pi / 3) \bmod \left(j_{z}, 3\right)$. Reminiscent of the flux (a) Sampled field

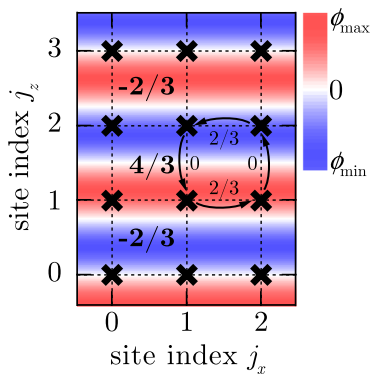

(b) Chern number

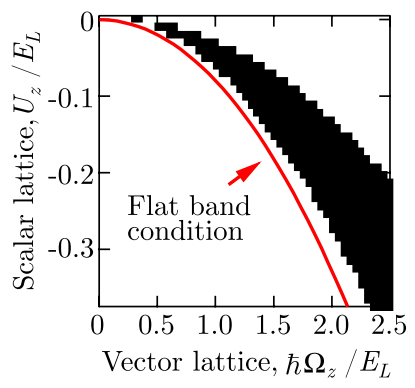

FIG. 5 (color online). Generation of the $1 / 3$ flux Hofstadter model. (a) Schematic showing effective $1 / 3$ flux per plaquette modulo 1 . The color scale indicates the effective phase gradient induced by the vector lattice. Atoms acquire phases as they hop along $\mathbf{e}_{x}$, in contrast, no phase is acquired by hopping along $\mathbf{e}_{z}$ (see loop). (b) Region (in black) where the Chern numbers in the lowest three bands are $(1,-2,1)$, equivalent to the $n_{\Phi}=1 / 3$ Hofstadter model as a function of the period $3 a / 2$ vector lattice's strength. The horizontal axis is its vector contribution to $\Omega_{z}$ and the vertical is its scalar contribution to the overall lattice potential. The inclusion of a state-dependent potential spatially modulates the energy in the lowest dressed band (including the effect of the scalar potential arising from the adiabatic approximation), resulting in an unwanted spatial staggering of the lattice potential. The flat band condition corresponds to the case when the scalar light shift cancels this unwanted modulation. 
rectification mechanism proposed in Ref. [22], this configuration can be created in our system by adding two standing waves along $\mathbf{e}_{z}$ (normal to the Raman lasers): a state-independent lattice with period $a$ localizing the atoms to specific lattice sites (e.g., from a retro-reflected $532 \mathrm{~nm}$ laser), and a state-dependent "vector" lattice with a period $3 a / 2$ sinusoidally modulating $\Omega_{z}$ as a function of $z$ (e.g., from an additional $790 \mathrm{~nm}$ laser, nearly counterpropagating). Figure 5(a) shows that with a suitable relative phase between the standing waves, the fluxes along $\mathbf{e}_{z}$ repeat with the pattern $(-2 / 3,4 / 3,-2 / 3)$, giving the desired flux per plaquette. To verify this heuristic interpretation, we numerically solve the 2D band structure (for exact parameters see [14]), and as shown in Fig. 5(b), we confirm that for a wide range of parameters, the three lowest bands are described by the same Chern numbers [23] as are those of the $n_{\Phi}=1 / 3$ Hofstadter model: $(1,-2,1)$.

We realized a 1D lattice potential for ultracold atoms using only rf and Raman transitions, in which the tunneling matrix element is, in general, complex. This work constitutes a first step toward realizing flux lattices [21], in which the physics of charged particles in strong magnetic fields can be simulated. The tunability of the Peierls phase achieved with our rf-Raman lattice would allow the observation of nonlinear effects of ultracold atoms in 1D periodic potentials, such as atomic density modulations with periodicity larger than the lattice spacing [24].

We appreciate enlightening conversations with G. Juzeliunas, N.R. Cooper, and W.D. Phillips. This work was partially supported by the ARO with funding from DARPA's OLE program and the Atomtronics-MURI, and the NSF through the JQI Physics Frontier Center. K. J.-G. thanks CONACYT, L. J.L. thanks NSERC, and M. C. B. thanks the NIST-ARRA program.

Note added in proof.-Recently we learned of a complementary experimental technique to manipulate the Peierls phase in driven optical lattices [25].

*ian.spielman@nist.gov

[1] J. Dalibard, F. Gerbier, G. Juzeliūnas, and P. Öhberg, Rev. Mod. Phys. 83, 1523 (2011).

[2] A. S. Sørensen, E. Demler, and M. D. Lukin, Phys. Rev. Lett. 94, 086803 (2005).

[3] M. Hafezi, A. S. Sørensen, E. Demler, and M. D. Lukin, Phys. Rev. A 76, 023613 (2007).

[4] R. Grimm, M. Weidemüller, and Y. B. Ovchinnikov, Adv. At. Mol. Opt. Phys. 42, 95 (2000).

[5] D. R. Hofstadter, Phys. Rev. B 14, 2239 (1976).
[6] Y.-J. Lin, R. L. Compton, K. Jiménez-García, J. V. Porto, and I. B. Spielman, Nature (London) 462, 628 (2009).

[7] D. Ciampini, O. Morsch, and E. Arimondo, Int. J. Quantum. Inform. 09, 139 (2011).

[8] R. A. Williams, S. Al-Assam, and C. J. Foot, Phys. Rev. Lett. 104, 050404 (2010).

[9] M. Aidelsburger, M. Atala, S. Nascimbène, S. Trotzky, Y.A. Chen, and I. Bloch, Phys. Rev. Lett. 107, 255301 (2011).

[10] M. V. Berry, Proc. R. Soc. A 392, 45 (1984).

[11] In some experiments, both the trap frequencies and $\lambda$ were slightly different: $\left(f_{x}, f_{y}, f_{z}\right)=(17.3,41.4,90) \mathrm{Hz}$ and $\lambda=790.14 \mathrm{~nm}$.

[12] Y.-J. Lin, R. L. Compton, A. R. Perry, W. D. Phillips, J. V. Porto, and I. B. Spielman, Phys. Rev. Lett. 102, 130401 (2009).

[13] Y.-J. Lin, R. L. Compton, K. Jiménez-García, W. D. Phillips, J. V. Porto, and I. B. Spielman, Nature Phys. 7, 531 (2011).

[14] See Supplemental Material at http://link.aps.org/ supplemental/10.1103/PhysRevLett.108.225303 for details on the technique to induce sloshing, the momentum representation of the rf-Raman Hamiltonian to calculate band structure and lattice properties, the deloading procedure, and the specific parameters for the proposed Hofstadter model.

[15] S. Chen et al., arXiv:1201.6018v1.

[16] Uncertainties reflect the uncorrelated combination of onestandard deviation statistical and systematic uncertainties.

[17] The general relation between the effective mass and tunneling amplitude is $m / m^{*}\left(k_{x}\right)=\pi^{2} \cos \left(\pi k_{x} / k_{L}-\right.$ $\phi) t / E_{L}$. For small sloshing amplitudes $\Delta k_{x}$ around the minimum $k_{x}=(\phi / \pi) k_{L}$, the effective mass is almost uniform.

[18] We use $\Omega_{\mathrm{rf}}=\Omega_{\mathrm{rf}_{0}}+\Delta \Omega_{\mathrm{rf}} \cos \left(2 \pi f_{\mathrm{rf}} \Omega_{z}\right)$, where $\hbar \Omega_{\mathrm{rf}_{0}}=$ $0.75 E_{L}, \hbar \Delta \Omega_{\mathrm{rf}}=0.23 E_{L}$, and $f_{\mathrm{rf}} / \hbar=0.4 E_{L}^{-1}$.

[19] S. Burger, F. S. Cataliotti, C. Fort, F. Minardi, M. Inguscio, M. L. Chiofalo, and M.P. Tosi, Phys. Rev. Lett. 86, 4447 (2001).

[20] Y.B. Ovchinnikov, J.H. Müller, M.R. Doery, E. J. D. Vredenbregt, K. Helmerson, S.L. Rolston, and W.D. Phillips, Phys. Rev. Lett. 83, 284 (1999).

[21] N. R. Cooper, Phys. Rev. Lett. 106, 175301 (2011).

[22] F. Gerbier and J. Dalibard, New J. Phys. 12, 033007 (2010).

[23] T. Fukui, Y. Hatsugai, and H. Suzuki, J. Phys. Soc. Jpn. 74, 1674 (2005).

[24] M. Machholm, A. Nicolin, C. J. Pethick, and H. Smith, Phys. Rev. A 69, 043604 (2004).

[25] J. Struck, C. Ölschläger, M. Weinberg, P. Hauke, J. Simonet, A. Eckardt, M. Lewenstein, K. Sengstock, and P. Windpassinger Phys. Rev. Lett. 108, 225304 (2012). 